\title{
La reproduction sociale dans les familles françaises des immigrants partis de l'île de Ré au Canada au XVII ${ }^{\mathrm{e}}$ siècle
}

\section{Claire Lambert et Yves Landry}

Volume 55, numéro 3, hiver 2002

URI : https://id.erudit.org/iderudit/010414ar

DOI : https://doi.org/10.7202/010414ar

Aller au sommaire du numéro

\section{Éditeur(s)}

Institut d'histoire de l'Amérique française

\section{ISSN}

0035-2357 (imprimé)

1492-1383 (numérique)

Découvrir la revue

\section{Citer cet article}

Lambert, C. \& Landry, Y. (2002). La reproduction sociale dans les familles françaises des immigrants partis de l'île de Ré au Canada au XVII ${ }^{\mathrm{e}}$ siècle. Revue d'histoire de l'Amérique française, 55(3), 345-379.

https://doi.org/10.7202/010414ar
Résumé de l'article

Cette étude cherche à cerner différents éléments ayant pu influencer la décision de 37 hommes et femmes de quitter la paroisse de Saint-Martin de l'île de Ré en direction du Canada au xviie siècle. Dans la majorité des cas, les émigrants et leurs familles, de souche martinaise, sinon rhétaise, avaient baigné dans un environnement social, économique et religieux spécifique, marqué notamment par la proximité avec le port de La Rochelle. En premier lieu, la société martinaise était profondément conditionnée par la monoculture de la vigne. Même si l'exploitation était le plus souvent de taille réduite et morcelée, les surfaces cultivées étaient en général suffisantes pour en vivre, d'autant que la pratique d'un travail annexe pouvait assurer des revenus complémentaires. Les familles des émigrants n'étaient nullement miséreuses, comme le démontre l'étude des apports au mariage. Mais, dans le but d'éviter un morcellement croissant de l'exploitation à la suite d'un partage égalitaire strict en contexte de saturation du terroir, les familles mirent en place des stratégies visant à sa préservation. Cette situation pouvait engendrer le départ de certains, les émigrants s'excluant alors eux-mêmes du partage successoral. En deuxième lieu, les futurs émigrants ont pu être sensibilisés aux voyages au long cours par suite de l'activité portuaire importante de Saint-Martin. Enfin, l'influence de La Rochelle sur l'île de Ré n'était pas seulement économique, mais également idéologique. Ce bastion de la Réforme véhiculait les idées protestantes auxquelles adhéra une partie de la population martinaise, dont quelques émigrants pour la colonie.
Tous droits réservés (C) Institut d'histoire de l'Amérique française, 2000

Ce document est protégé par la loi sur le droit d'auteur. L'utilisation des services d'Érudit (y compris la reproduction) est assujettie à sa politique d'utilisation que vous pouvez consulter en ligne.

https://apropos.erudit.org/fr/usagers/politique-dutilisation/ 


\title{
La reproduction sociale dans les familles françaises des immigrants partis de l'île de Ré au Canada au XVII ${ }^{\mathrm{e}}$ siècle $^{\mathrm{I}}$
}

\author{
CLAIRE LAMBERT et YVES LANDRY \\ Programme de recherche sur l'émigration des Français \\ en Nouvelle-France (PRÉFEN) \\ Centre de recherche d'histoire quantitative \\ Université de Caen Basse-Normandie
}

RÉSUMÉ • Cette étude cherche à cerner différents éléments ayant pu influencer la décision de 37 hommes et femmes de quitter la paroisse de Saint-Martin de l'île de Ré en direction du Canada au XVII ${ }^{\mathrm{e}}$ siècle. Dans la majorité des cas, les émigrants et leurs familles, de souche martinaise, sinon rhétaise, avaient baigné dans un environnement social, économique et religieux spécifique, marqué notamment par la proximité avec le port de La Rochelle. En premier lieu, la société martinaise était profondément conditionnée par la monoculture de la vigne. Même si l'exploitation était le plus souvent de taille réduite et morcelée, les surfaces cultivées étaient en général suffisantes pour en vivre, d'autant que la pratique d'un travail annexe pouvait assurer des revenus complémentaires. Les familles des émigrants n'étaient nullement miséreuses, comme le démontre l'étude des apports au mariage. Mais, dans le but d'éviter un morcellement croissant de l'exploitation à la suite d'un partage égalitaire strict en contexte de saturation du terroir, les familles mirent en place des stratégies visant à sa préservation. Cette situation pouvait engendrer le départ de certains, les émigrants s'excluant alors eux-mêmes du partage successoral. En deuxième lieu, les futurs émigrants ont pu être sensibilisés aux voyages au long cours par suite de l'activité portuaire importante de Saint-Martin. Enfin, l'influence de La Rochelle

1. Cette recherche a été réalisée avec l'aide financière du Conseil de recherches en sciences humaines du Canada (programme de subventions ordinaires de recherche). Les travaux du PRÉFEN bénéficient de l'appui du ministère du Patrimoine canadien, de l'Université de Caen Basse-Normandie, du CNRS, du Conseil général de l'Orne et de la Communauté de communes du Haut-Perche. Les auteurs remercient également les Archives départementales de la CharenteMaritime qui ont aimablement communiqué l'illustration du port de Saint-Martin de l'île de Ré reproduite en couverture. 
sur l'île de Ré n'était pas seulement économique, mais également idéologique. Ce bastion de la Réforme véhiculait les idées protestantes auxquelles adhéra une partie de la population martinaise, dont quelques émigrants pour la colonie.

ABSTRACT - This article examines different factors which may have influenced the decision of 37 men and women to leave the parish of St. Martin in the Island of Ré to go to Canada during the $\mathrm{XVII}^{\text {th }}$ century. For the majority of them, the emigrants and their families originated from St. Martin, if not from the Island of Ré, and lived in a specific social, economic, and religious environment, influenced in particular by the proximity of La Rochelle. Many factors may have played in favor of emigration. Firstly, St. Martin lived from wine cultivation. The average family wineyard being small but still profitable, it was important not to diminish its size through a strict equalitarian sharing among the heirs. Some compensatory strategies were used in order to preserve the integrity of the land, and these strategies may have encouraged children to emigrate. Secondly, many among the future emigrants may have been familiar with transoceanic voyages, since St. Martin was an important harbour. And lastly the religious influence of La Rochelle must be taken into consideration. This strong hold of the Reform spread many Protestant ideas to which part of the population from St. Martin adhered, including some emigrants to the New World.

ES RECHERCHES menées au Québec depuis une trentaine d'années,
principalement en démographie historique, ont permis de mieux saisir le processus d'acculturation démographique des immigrants établis au Canada au $\mathrm{xvII}^{\mathrm{e}}$ siècle, c'est-à-dire de reconstituer le schéma par lequel les Français transplantés dans la colonie adoptèrent un comportement essentiellement caractérisé par une fécondité plus élevée et une mortalité moins forte qu'en métropole ${ }^{2}$. Au-delà de cet acquis fondamental, cependant, notre connaissance des origines sociales de ces immigrants a

2. Hubert Charbonneau, Bertrand Desjardins, André Guillemette, Yves Landry, Jacques Légaré et François Nault, Naissance d'une population. Les Français établis au Canada au XVII siècle (Montréal/Paris, Presses universitaires de France/Presses de l'Université de Montréal, 1987), viii232 p. Yves Landry, Orphelines en France, pionnières au Canada: les Filles du roi au XVII e siècle (Montréal, Leméac, 1992), 436 p. Jean-Pierre Bardet et Hubert Charbonneau, "Cultures et milieux en France et en Nouvelle-France. La différenciation des comportements démographiques», dans Joseph Goy et Jean-Pierre Wallot, dir., Évolution et éclatement du monde rural : structures, fonctionnement et évolution différentielle des sociétés rurales françaises et québécoises, $\mathrm{XVII}^{e}$-XX ${ }^{e}$ siècles (Paris / Montréal, Éditions de l'École des hautes études en sciences sociales/Presses de l'Université de Montréal, 1986), 75-88. Yves Landry, «Fertility in France and New France: The Distinguishing Characteristics of Canadian Behavior in the Seventeenth and Eighteenth Centuries", Social Science History, 17,4 (hiver 1993) : 577-592. Pour un bilan critique de l'historiographie sur les immigrants au Canada sous le Régime français, voir Yves Landry, «Les immigrants en Nouvelle-France : bilan historiographique et perspectives de recherche», dans Philippe Joutard et Thomas Wien, dir., Mémoires de Nouvelle-France (Rennes/Sillery, Presses universitaires de Rennes/Septentrion, 2002), à paraître. 
progressé de façon assez limitée, si l'on excepte le portrait descriptif que l'on peut tirer de leurs déclarations contenues dans les sources canadiennes et qui nous renseigne peu sur leurs antécédents français ${ }^{3}$.

Heureusement, une enquête prosopographique menée depuis quelques années dans les archives françaises, totalisant à ce jour près de quatre cents essais biographiques, permet de mieux comprendre les facteurs sous-jacents à l'acte migratoire ${ }^{4}$. Le présent travail, qui porte sur un groupe de 37 hommes et femmes immigrés au Canada au XviI ${ }^{\mathrm{e}}$ siècle, s'intègre dans le cadre de cette enquête collective. Tous originaires de la paroisse de Saint-Martin de l'île de Ré, en la province de l'Aunis (département actuel de la Charente-Maritime), ceux-ci sont partis sur des navires mouillant à La Rochelle, principal port d'embarquement pour la colonie avant 1740, situé à quelques kilomètres de Ré.

L'échantillon se compose de 23 hommes et 14 femmes, dont les trois quarts étaient âgés de moins de 25 ans à leur première mention dans les sources canadiennes. Comme l'indique le tableau 1, leur mode de recrutement, comme engagés, «Filles du roi» ou militaires, est connu dans moins de quatre cas sur dix.

À l'instar de l'ensemble des immigrants français du XviI ${ }^{\mathrm{e}}$ siècle ${ }^{5}$, les Martinais ont rarement émigré de façon isolée. Quatorze d'entre eux, soit plus du tiers, appartenaient à trois familles nucléaires : deux étaient formées d'un couple et de quatre enfants âgés de un à seize ans; l'autre se composait d'un père accompagné de sa fille. Dix autres immigrants devaient entretenir entre eux des liens d'amitié ou du moins de connaissance : les trois engagés de 1664 embarqués sur Le navire Noir de Hollande, soit François Marchand et les protestants Jacques Baudoin et Nicolas Geoffroy; les trois engagés de 1665, Pierre Neveu, Pierre Mercereau et Nicolas Butaud, tous célibataires, âgés de moins de vingt ans, embarqués sur Le Cat de Hollande et contractants auprès du marchand rochelais Pierre Gaigneur, les deux premiers le 31 mars 1665, l'autre quatre jours plus tard; les deux soldats du régiment de Carignan-Salières, Nicolas

3. Jean-Pierre Poussou a critiqué la minceur du bilan des origines sociales des immigrantes établies au Canada au XviI ${ }^{\mathrm{e}}$ siècle dans Annales de démographie historique (1993) : 435.

4. Yves Landry, «L'émigration française au Canada avant 1760 : premiers résultats d'une microanalyse», dans Andrée Courtemanche et Martin Pâquet, dir., Prendre la route. L'expérience migratoire en Europe et en Amérique du Nord du XIV $v^{e}$ au xx $x^{e}$ siècle (Hull, Éditions Vents d'Ouest, 2001), 81-105.

5. André Guillemette et Jacques Légaré, «The Influence of Kinship on Seventeenth-Century Immigration to Canada ", Continuity and Change, 4,1 (février 1989) : 79-102. 
TAB LEA U

Année de première mention dans les sources canadiennes, mode de recrutement et statut familial à l'arrivée des immigrants martinais

\begin{tabular}{|c|c|c|c|c|c|c|c|c|}
\hline \multirow[b]{2}{*}{ Année } & \multicolumn{3}{|c|}{ Mode de recrutement connu } & \multicolumn{4}{|c|}{ Mode de recrutement inconnu } & \multirow[b]{2}{*}{ Total } \\
\hline & $\begin{array}{c}\text { Engagé } \\
\text { célibataire }\end{array}$ & $\begin{array}{c}\text { Militaire } \\
\text { célibataire }\end{array}$ & $\begin{array}{l}\text { Fille } \\
\text { du roi }\end{array}$ & $\begin{array}{c}\text { Couple } \\
\text { avec enfants }\end{array}$ & $\begin{array}{c}\text { Père } \\
\text { avec enfant }\end{array}$ & $\begin{array}{l}\text { Homme } \\
\text { célibataire }\end{array}$ & $\begin{array}{c}\text { Femme } \\
\text { célibataire }\end{array}$ & \\
\hline 1657 & \multirow{12}{*}{$\begin{array}{l}3^{*} \\
3^{*}\end{array}$} & \multirow{12}{*}{$2 *$} & \multirow{4}{*}{1} & \multirow{12}{*}{12} & \multirow{12}{*}{2} & 1 & \multirow{12}{*}{1} & 1 \\
\hline 1663 & & & & & & 1 & & 2 \\
\hline 1664 & & & & & & \multirow{5}{*}{1} & & 6 \\
\hline 1665 & & & & & & & & 6 \\
\hline 1666 & & & 1 & & & & & 1 \\
\hline 1669 & & & 3 & & & & & 15 \\
\hline 1672 & & & 1 & & & & & 1 \\
\hline 1677 & & & & & & 1 & & 1 \\
\hline 1700 & & & & & & 1 & & 1 \\
\hline 1705 & & & & & & 1 & & 1 \\
\hline 1706 & & & & & & 1 & & 1 \\
\hline 1710 & & & & & & 1 & & 1 \\
\hline Total & 6 & 2 & 6 & 12 & 2 & 8 & 1 & 37 \\
\hline
\end{tabular}

* Il s'agit de l'année précise d'arrivée. 
Bonin et André Mignier; enfin, les deux Filles du roi, Marie Jalais et Barbe Ménard, qui se marièrent à la même date à Sainte-Famille de l'île d'Orléans. Seuls 13 immigrants, soit le tiers, ne présentent pas de liens de solidarité connus.

L'intérêt principal de cette recherche réside dans la tentative de reconstituer la vie des immigrants et de leur famille dans leur milieu d'origine, mettant ainsi en lumière les facteurs qui, dans un contexte économique et politique ne favorisant pourtant pas le peuplement du Canada, ont pu conditionner et générer leur départ. L'historiographie québécoise a déjà cherché à expliquer la minceur du flux migratoire entre France et Nouvelle-France - environ 30000 immigrants en 150 ans $^{6}$ - et l'impuissance du Canada à transformer ses visiteurs en colons. Elle avance le fait que la jeune colonie n’offrait pas les structures économiques nécessaires pour attirer et conserver son capital humain. L'économie fondée sur le commerce des fourrures exigeait en effet peu de main-d'œuvre, en dehors des Amérindiens pour la cueillette des pelleteries et leur transport au comptoir de traite, ainsi que de quelques commis et interprètes pour assurer la bonne marche des opérations ${ }^{7}$. Mais trois facteurs propres à la France d'Ancien Régime complètent cette première explication. Tout d'abord, à l'inverse de leurs voisins ibériques et anglais, qui ont abondamment peuplé leurs colonies américaines, les Français quittaient rarement le sol du royaume. Leur incontestable sédentarité aux $\mathrm{xvII}^{\mathrm{e}}$ et $\mathrm{xVIII}^{\mathrm{e}}$ siècles, la plus généralisée et la plus persistante de toute l'histoire de la population française, a non seulement réduit à peu de chose le nombre de départs à l'étranger, mais a même conféré un statut minoritaire - sauf exceptions locales ou conjoncturelles - aux migrations intérieures, qu'elles fussent saisonnières, temporaires ou définitives. D’après Pierre Goubert, environ un million de Français changeaient provisoirement ou définitivement de domicile, chaque année, à courte comme à longue distance. Seulement $5 \%$ des Français étaient donc mobiles en temps normal. Parmi

6. Mario Boleda, "Trente mille Français à la conquête du Saint-Laurent ", Histoire sociale/Social History, 23,45 (mai 1990) : 153-177. Le même auteur a récemment révisé à la hausse ce nombre d'immigrants, qui aurait dépassé 33 500. Mario Boleda, «Nouvelle estimation de l’immigration française au Canada, 1608-1760", dans Yves Landry, dir., Actes des Premières Journées d'étude du PRÉFEN. Le peuplement du Canada aux XVII et XVIII ${ }^{e}$ siècles. Stratégie d'une recherche réalisée dans le cadre de la Maison de l'émigration française en Canada (Caen, Centre de recherche d'histoire quantitative, Université de Caen Basse-Normandie, 2002), à paraitre.

7. John A. Dickinson, "Les Amérindiens et les débuts de la Nouvelle-France», dans Luca Codignola et Raimondo Luraghi, dir. Canada ieri e oggi. Atti del $6^{\circ}$ Convegno internazionale di studi canadesi, Selva di Fasano, 27-31 marzo 1985, (Schena Editore, 1986), III : 92-93. 
eux, environ 100000 traversaient les frontières sous forme de migrations saisonnières et temporaires, tandis qu'entre 15000 et 30000 gagnaient définitivement un pays voisin ou les colonies, soit approximativement 1 Français sur $1000^{8}$.

Un autre facteur de la faiblesse du mouvement migratoire vers le Canada est l'absence de volonté réelle de l'État à promouvoir l'émigration vers le Nouveau Monde. Le fondement doctrinaire et politique de cette attitude est bien connu : on croyait que le royaume se dépeuplait. Moheau a déjà témoigné de cette appréhension commune depuis le xvI siècle en dénonçant le fait qu' "en France l'expatriation soit une maladie nationale ${ }^{9}$. Cette fausse impression découlait de ce qu'il y avait bien plus de Français à l'étranger que d'étrangers en France. Les autorités n'ont donc pas cherché à accentuer l'hémorragie dont on croyait la France victime en forçant l'émigration vers les colonies.

Un dernier facteur d'inertie migratoire est le refus obstiné d'autoriser l'établissement de protestants au Nouveau Monde. Les historiens, Garneau et Salone en tête ${ }^{10}$, ont bien vu que cette interdiction signifiait la fermeture de «l'Amérique française à ceux-là même des Français qui avaient le plus d'intérêt à chercher outre-mer une patrie nouvelle ». Toutefois, les archives canadiennes signalent la présence d'au moins 850 personnes qui auraient été protestantes à un moment ou à un autre de leur vie ${ }^{11}$. Originaires de la France, de l'Angleterre et des colonies américaines, plusieurs d'entre elles se sont installées au Canada en dépit des barrières élevées contre elles. Dans la plupart des cas, leur intégration a été facilitée par leur abjuration tacite ou formelle. De récents travaux évaluent même à au moins $6 \%$ à $8 \%$ la proportion de protestants dans l'ensemble de l'immigration au Canada avant 1760, soit environ 2000 per-

8. Communication personnelle de Jean-Pierre Poussou. Le démographe Henri Bunle a déjà estimé le nombre annuel de départs pour l'étranger à 12 000. Henri Bunle, Mouvements migratoires entre la France et l'étranger (Paris, Imprimerie nationale, Service national des statistiques, coll. «Études démographiques», nº 4, 1943), 20. Voir aussi Jean-Pierre Poussou, «Mobilité et migrations», dans Jacques Dupâquier, dir., Histoire de la population française, 2 : De la Renaissance à 1789 (Paris, Presses universitaires de France, 1988), 99.

9. Jean-Baptiste Moheau, Recherches et Considérations sur la population de la France (1778) (Paris, Éditions de l’Institut national d'études démographiques, 1994), 185. Réédition annotée par Éric Vilquin.

10. François-Xavier Garneau, Histoire du Canada depuis sa découverte jusqu'à nos jours (Québec, Lovell, 1852), 1:71. Émile Salone, La colonisation de la Nouvelle-France. Étude sur les origines de la nation canadienne-française (Paris, Guilmoto, 1905), 44-45.

11. Marc-André Bédard, Les protestants en Nouvelle-France (Québec, Société historique du Québec, 1978), 141 p. 
sonnes ${ }^{12}$. Malgré cette réalité occultée par l'historiographie traditionnelle, on ne saurait cependant assimiler le Canada aux Antilles où une politique plus tolérante envers les protestants était appliquée, en dépit de la stricte volonté royale de les exclure ${ }^{13}$.

Tous ces facteurs de restriction migratoire relèvent principalement de la macro-analyse historique, dont la pertinence pour expliquer les motivations des migrants a déjà montré ses limites ${ }^{14}$. Si l'on veut s'affranchir du niveau descriptif auquel ont conduit les études menées jusqu'à maintenant sur les aires migratoires et la conjoncture socio-économique, il faut résolument adopter une approche micro-analytique et rechercher les causes de l'émigration vers la Nouvelle-France au sein de chaque famille migrante ${ }^{15}$. C'est en se penchant en effet sur les migrants eux-mêmes, jusque dans leur histoire personnelle et familiale, que l'on pourra tenter d'approcher au mieux les raisons qui ont pu les inciter à partir pour une contrée nouvelle dont la réputation inhospitalière, due principalement aux hivers rudes et aux Iroquois, a traversé l'Atlantique avec le trafic maritime et les marins jamais avares de récits audacieux ${ }^{16}$.

12. Robert Larin, Brève histoire des protestants en Nouvelle-France et au Québec (XVI $I^{e}$-XIX ${ }^{e}$ siècles) (Saint-Alphonse-de-Granby, Éditions de la Paix, 1998), 206 p. Seules de nouvelles recherches menées dans les archives françaises permettront éventuellement de mieux quantifier et de mieux caractériser cet apport protestant. Sur les travaux récemment menés sur les immigrants catholiques et protestants partis au xvII ${ }^{\mathrm{e}}$ siècle du Moyen-Poitou, voir Claire Lambert, Catholiques et protestants du Moyen-Poitou vers la Nouvelle-France au XVII ${ }^{e}$ siècle, mémoire de DEA (histoire), Université d'Artois, 2000, 394 p.

13. Jacques Petitjean-Roget, "Les protestants à la Martinique sous l'Ancien Régime», Revue d'histoire des colonies, 42 ( $2^{\mathrm{e}}$ trimestre 1955) : 220-265. Lucien-René Abenon, «La survie de la communauté protestante à la Martinique et à la Guadeloupe au xvIII ${ }^{\mathrm{e}}$ siècle », dans Guy Martinière, Didier Poton et François Souty, dir., D'un rivage à l'autre. Villes et protestantisme dans l'aire atlantique (XVI-XVII ${ }^{e}$ siècles) (Paris, Imprimerie nationale, 1999), 251-256.

14. Voir par exemple Robert Mandrou, "Les Français hors de France aux xvI ${ }^{\mathrm{e}}$ et XvII ${ }^{\mathrm{e}}$ siècles", Annales ESC, 14,4 (octobre-décembre 1959) : 662-675.

15. C'est l'approche prônée entre autres par Gabriel Debien et Jean-Pierre Poussou. Gabriel Debien, «L'émigration poitevine vers l'Amérique au xvII siècle», Bulletin de la Société des Antiquaires de l'Ouest et des Musées de Poitiers, II (1952) : 305-306. Lucile Bourrachot et Jean-Pierre Poussou, "L'émigration quercynoise par le port de Bordeaux au XvirI siècle», Bulletin de la Société des Études $d u$ Lot, 95 (1974) : 2. Jean-Pierre Poussou, «Migrations et mobilité de la population en Europe à l'époque moderne", dans Jean-Pierre Bardet et Jacques Dupâquier, dir., Histoire des populations de l'Europe, I: Des origines aux prémices de la révolution démographique (Paris, Fayard, 1997), 284.

16. Sur ce problème de l'image du Canada transmise par les voyageurs et dans les imprimés, voir Yves Landry, «Les Français passés au Canada avant 1760 : le regard de l'émigrant», dans Jean-Pierre Bardet, dir., Français et Québécois : le regard de l'autre (Paris, Centre de coopération interuniversitaire franco-québécoise, 2002), à paraitre. 
Ainsi avons-nous cherché à reconstituer, essentiellement à l'aide des registres paroissiaux catholiques ou protestants et des minutes des notaires résidant à Saint-Martin, la situation familiale, économique et religieuse des 37 émigrants identifiés grâce aux déclarations d'origine consignées dans les sources canadiennes et grâce à divers travaux généalogiques ${ }^{17}$. Deux obstacles ont cependant gêné notre effort. D’abord, les nombreuses lacunes des sources martinaises, puisque les registres paroissiaux des deux confessions ont disparu dans l'incendie de la Mairie de Saint-Martin en 1891; heureusement, une copie partielle en avait été faite avant le sinistre par un érudit local, René-Théodore Phelippot, transcription que nous avons utilisée à défaut des registres originaux et qui a permis de reconstituer au moins en partie l'ascendance de la plupart des immigrants. Les minutiers des notaires de Saint-Martin souffrent aussi de déficiences importantes, attribuables à des pertes accidentelles et à diverses négligences ${ }^{18}$. Second obstacle : la difficulté de repérer tous les Martinais émigrés au Canada. Il est fort probable qu'une fraction d'entre eux nous échappe du fait qu'ils n'ont pas laissé de trace dans les archives canadiennes ${ }^{19}$. Cette limite à l'intégralité de la population étudiée est cependant inévitable et rien ne permet de croire que les immigrants connus ne sont pas représentatifs de l'ensemble.

Nous nous proposons donc de présenter ici les principaux aspects de la situation de ces immigrants avant leur départ, en abordant notamment la place de leurs familles dans cette paroisse rhétaise et les stratégies mises en œuvre pour tenter de préserver leur patrimoine. Nous évoquerons également l'influence non négligeable de la situation géographique de l'île, en relation étroite avec le commerce international et le port voisin de La Rochelle.

17. René Jetté, Dictionnaire généalogique des familles du Québec : des origines à 1730 (Montréal, Presses de l'Université de Montréal, 1983), 1176 p. Normand Robert, Nos origines en France, des débuts à 1825, 4 : L'Aunis (Montréal, Archiv-Histo, 1988), 78 p. André Guillemette, "L'émigration rhétaise en Nouvelle-France", Mémoires de la Société généalogique canadienne-française, 42,1 (printemps 1991) : 43-51. Fédération québécoise des sociétés de généalogie et Fédération française de généalogie, Fichier Origine (sur Internet). Ont été considérés les immigrants ayant déclaré comme lieux d'origine Saint-Martin, Le Bois ou La Couarde - ces deux derniers villages, annexes de la paroisse de Saint-Martin jusqu’à la veille de la Révolution - , ainsi que ceux rattachés simplement à l' "île de Ré», dans la perspective de les retrouver éventuellement à Saint-Martin.

18. Pour une critique complète des sources, voir Claire Lambert, Une contribution de l'île de Ré au peuplement de la Nouvelle-France : la paroisse de Saint-Martin au XVII siècle, mémoire de maîtrise (histoire), Université de La Rochelle, 1999, 8-13.

19. Si les Martinais reflètent la situation générale des immigrants français au Canada avant 1760, seulement la moitié d'entre eux seraient connus nominativement par des mentions dans les registres paroissiaux. 


\section{UN CONTINGENT ISSU D'UNE PAROISSE RURALE VITICOLE}

Une première caractéristique essentielle du contingent des Martinais émigrés en Nouvelle-France concerne leurs origines proprement rhétaises.

\section{Enracinement des familles d'immigrants dans l'île de Ré}

On a pu établir, au terme de l'exploitation des registres paroissiaux et des actes notariés de la paroisse de Saint-Martin, que plus de $81 \%$ des familles d'immigrants avaient des origines rhétaises. Cette proportion rassemble les cas où les grands-parents paternels ou maternels étaient présents dans l'île et où une mention d'origine rhétaise était portée dans l'acte de mariage ou de remariage d'au moins un des parents de l'immigrant ou encore dans l'acte de baptême de ce dernier dans la paroisse de Saint-Martin (tableaux 2 et 3 ).

Ainsi, plus de la moitié du contingent étudié peut attester d'aïeux dans l'île. Nous avons même pu retrouver la trace des arrière-grands-parents de certains émigrants, comme ceux de Françoise Asselin, Nicolas Bonin et Mathieu Guillet, voire celle de l'arrière-arrière-grand-mère de ce dernier. À l'intérieur de la paroisse de Saint-Martin, le village de La Couarde a accueilli au moins trois générations en ligne directe de Bonin et le hameau du Morinand, quatre générations de Guillet. Et même si le grand-père paternel de Françoise Asselin, du bourg de Saint-Martin, s'est établi à quelques kilomètres de ce lieu, dans le bourg d'Ars, en raison de sa profession de procureur des seigneuries d'Ars et de Loix, son fils Gilles revint plus tard à Saint-Martin en tant que marchand.

Cet enracinement spatial confirme dans un premier temps le peu de goût de cette population pour les déplacements, qui ne s'effectuaient généralement que sur un territoire très limité. Cela n'avait rien d'exceptionnel en cette France du xvir ${ }^{\mathrm{e}}$ siècle où, selon Jean-Pierre Poussou, "presque tous ceux nés dans une paroisse rurale pass[ai]ent leur existence

TABLEAU 2

Origine des immigrants

\begin{tabular}{l|c|c}
\hline & Nombres absolus & Nombres relatifs \\
\hline Ascendance reconnue à l'île de Ré & 30 & 81 \\
Immigrant présent à Saint-Martin & 3 & 8 \\
Aucune mention & 4 & 11 \\
\hline Total & 37 & 100 \\
\hline
\end{tabular}


TABLEAU 3

Degré d'ascendance des immigrants rhétais

\begin{tabular}{l|c|c}
\hline & Nombres absolus & Nombres relatifs \% \\
\hline Parents et Grands-parents connus & 20 & 54 \\
Parents connus seulement & 10 & 27 \\
\hline Total & 30 & 81 \\
\hline
\end{tabular}

entière dans cette paroisse ou dans un cadre ne dépassant pas 10 kilomètres de rayon [et où il en allait] de même pour les bourgs ou les toutes petites villes ${ }^{20}$ ».

Cette observation sur la sédentarité des familles des immigrants de SaintMartin diverge cependant des conclusions tirées des autres études réalisées en France sur l'immigration au Canada avant 1760, comme celle de Benoît Poupart relative à la petite ville de Hiers-Brouage située à une trentaine de kilomètres au sud de La Rochelle, lieu de départ de 47 immigrants dont seulement 22 y sont véritablement nés, ou encore celle de Leslie Choquette qui a exploité un échantillon imposant de 16000 personnes $^{21}$. Tous s'accordent sur l'hypothèse que l'immigration vers la Nouvelle-France serait le résultat d'une «tradition migratoire» familiale, Brouage par exemple n'étant que la dernière étape des immigrants sur le sol métropolitain avant de s'embarquer pour la colonie. La migration entraînerait alors d'autres migrations, sans qu'on puisse rattacher cette population en mouvement continu à un lieu d'existence véritable. Cette hypothèse contribuerait à expliquer pourquoi, en sus des raisons liées à l'état des sources, le taux de succès atteint jusqu'à maintenant pour retrouver en France les familles des futurs émigrants ne dépasse guère en moyenne $50 \%$.

L'enracinement local de la plupart des futurs émigrants de la paroisse de Saint-Martin, contrairement aux résultats des études antérieures, démontre bien l'hétérogénéité des diverses situations, même dans un contexte d'urbanisation similaire, et la nécessité de multiplier les analyses à l'échelle micro-historique, notamment dans les campagnes, moins étu-

20. Jean-Pierre Poussou, «Mobilité et migrations», loc. cit., 99.

21. Benoît Poupart, De Brouage en Canada, attraction urbaine et émigration coloniale au XVII siècle, mémoire de maîtrise (histoire), Université de La Rochelle, 1997, 220 p. Leslie Choquette, De Français à paysans. Modernité et tradition dans le peuplement du Canada français (Sillery/Paris, Septentrion/Presses de l'Université de Paris-Sorbonne, 2001), xi-325 p. Voir aussi Yves Landry, «L'émigration française au Canada avant 1760 : premiers résultats d'une microanalyse », loc. cit., $82-85$. 
diées jusqu'à maintenant que les petites et moyennes villes (Fontenay-leComte, Marans, Saint-Jean-d'Angély, Versailles ${ }^{22} \ldots$...

Cependant, malgré la stabilité de sa population, l'île de Ré était loin de vivre en autarcie et les rencontres ne manquaient jamais dans un port actif comme celui de Saint-Martin. L'expérience de l'émigration de certains individus vers la Nouvelle-France pouvait se juxtaposer, à l'intérieur des mêmes familles - par exemple celles de Jacques Baudoin et de Marie Langlois -, à celle de l'exil d'autres aux Antilles, tandis que le reste du noyau familial restait sur place. Le constat du binôme émigration/sédentarité nous interroge donc sur les raisons d'une telle sédentarité des familles d'émigrants martinais, afin de mieux comprendre ce qui a pu pousser certains de leurs membres à inventer leur avenir en terre d'Amérique.

Deux éléments paraissent déterminants dans l'enracinement fondamental de la population rhétaise : l'institution de privilèges économiques aux insulaires, afin de s'assurer le contrôle de l'île et de sa population, et la quasi-monoculture de la vigne instaurée dès la période médiévale. Le premier élément s'explique par la situation géographique de Ré : ses côtes basses et sablonneuses en faisaient un lieu propice aux débarquements et la proximité du continent rendait sa possession indispensable pour qui voulait faire la guerre à toute place forte du littoral aunisien. L'intérêt stratégique de l'île s'imposa au Moyen-Âge, dès le milieu du XII ${ }^{\mathrm{e}}$ siècle, et ses seigneurs féodaux durent imaginer une solution pour conserver cette position très convoitée face à la nouvelle place forte de La Rochelle. Selon René James ${ }^{23}$, cette solution consista à peupler suffisamment l'île pour qu'elle organisât elle-même sa défense sous la forme de milices, ce qui assurait aux seigneurs la possession de l'île sans besoin d'y entretenir de coûteuses troupes.

Dans le but de favoriser une immigration massive dans l'île et de s'assurer de la fidélité de ses habitants, les différents seigneurs, puis les rois d'Angleterre - qui s'en rendaient parfois maitres - et les rois de France concédèrent jusqu'à la veille de la Révolution une longue suite de chartes et de privilèges ${ }^{24}$. En février 1652, Louis XIv confirma les privilèges qui visaient, entre autres, «l'exemption et décharge de toutes tailles, taillons,

22. De ces quatre villes, seule celle de Versailles comptait cependant plus de 10000 habitants vers 1700 ou 1750. Bernard Lepetit, Les villes dans la France moderne (1740-1840) (Paris, Albin Michel, 1988), 450-452.

23. René James, Chartes et privilèges royaux de l'île de Ré (Paris, Sirey, 1939), 160 p.

24. Eugène Kemmerer, Histoire de l'île de Ré depuis les premiers temps historiques jusqu'à nos jours (La Rochelle, G. Mareschal, 1868), $1:$ 248-270. 
aides, subsides, subventions et tous autres droits, charges et subsides généralement quelconques ${ }^{25}$ ». En plus de la taille, les Rhétais étaient exemptés d'impôts indirects et connaissaient un régime douanier très particulier : alors que La Rochelle avec le Poitou faisait partie des «cinq grosses fermes» et que la Saintonge était "réputée étrangère ", Ré était considérée comme "pays étranger effectif", en principe interdit aux employés des fermes et complètement séparé du royaume, hormis un hommage de fidélité politique et un rattachement judiciaire au parlement de Paris ${ }^{26}$.

De la volonté des maîtres de l'île pour asseoir leurs prérogatives à l'intérieur de cette communauté est donc né un mouvement d'immigration remontant à la période féodale. Ces privilèges ont constitué en outre un élément déterminant pour sédentariser une partie importante de la population. En effet, pourquoi s'expatrier d'une terre où le seigneur et plus tard le roi faisaient tout pour avantager les habitants et s'efforcer de les retenir? Il faut donc voir là un facteur essentiel de l'enracinement des familles rhétaises qui complète l'explication classique d'une France largement sédentaire.

Un second facteur de sédentarisation réside dans la monoculture de la vigne dans cette partie de l'île. En effet, les seigneurs de Ré ont encouragé très tôt l'implantation de la vigne pour mettre en valeur les terres de la baronnie. Comme la viticulture nécessitait une main-d'œuvre nombreuse en raison des fréquentes opérations culturales exigées, elle ne pouvait que contribuer à augmenter encore le nombre des défenseurs du territoire. Ce développement fut d'autant plus encouragé que la proximité de voies navigables avec le port de Saint-Martin favorisait l'implantation de ces vignobles commerciaux et que l'absence de paiement de droit de sortie facilitait l'exportation du vin rhétais sur les marchés étrangers.

L'étude du milieu professionnel des futurs émigrants montre que celuici était majoritairement lié à la viticulture et au négoce. La compilation présentée au tableau 4 repose sur les déclarations de profession de leur père, le jeune âge des immigrants et leur statut de célibataires ne leur ayant pas permis de faire apparaitre des mentions de profession dans les sources martinaises.

L'activité portuaire de Saint-Martin et la viticulture semblent avoir défini dans une large mesure la structure professionnelle de l'île. Les terres de la baronnie apparaissent en effet, au xvII ${ }^{\mathrm{e}}$ siècle, couvertes de vignes

25. Georges Musset, Les ports francs : étude historique (Paris, Leroux, 1904), 33.

26. René James, op. cit., 117. 
TABLEAU 4

Professions des pères des immigrants

\begin{tabular}{l|c}
\hline \multicolumn{1}{c|}{ Profession } & Nombres absolus \\
\hline Tonnelier & 14 \\
Laboureur & 5 \\
Tonnelier et laboureur & 2 \\
Marchand & 3 \\
Artisan & 3 \\
Soldat & 1 \\
«Hostelier» & 1 \\
Marinier & 1 \\
Inconnue & 7 \\
\hline Total & 37 \\
\hline
\end{tabular}

de Sablanceaux à La Couarde, ce que constate Louis Arcère pour le milieu du siècle suivant ${ }^{27}$. Mais d'autres indices attestent aussi l'importance du travail de la vigne ou du métier de vigneron. D’abord, le nombre de celliers ou de ce qui était sommairement appelé «dépendances», cités dans les contrats de partage, comme celui de l'arrière-grand-père de Mathieu Guillet $^{28}$, ou dans les inventaires de biens après décès, comme celui du père de Jacques Baudoin ${ }^{29}$. Les sources ne mentionnent pas de vin dans les celliers des petits propriétaires puisque la vendange et le vin nouveau étaient vendus aux négociants et les viticulteurs n'en gardaient que très peu pour la consommation familiale. Ensuite, les logis en pays de vignoble dominant étaient pour la plupart moins sommaires que sur des terres vouées, par exemple, à la culture céréalière. Enfin, l'omniprésence de la vigne sur le territoire est encore révélée dans les minutes martinaises par l'utilisation du «cep» comme unité de mesure pour une terre qui n'était pas forcément destinée à la vigne, par exemple un bois ou une cour. Ainsi, Mathieu Guillet, l'arrière-grand-père du futur émigrant Mathieu Guillet, fit le partage de ses biens en trois lots le 2 janvier 1655; son fils, Maurice

27. Louis Arcère, Histoire de la ville de La Rochelle et du Pays d'Aulnis (La Rochelle, Desbordes, 1756-1757), réimpression Marseille, 1975, 2 vol.

28. Archives départementales de la Charente-Maritime (désormais ADCM), contrat de partage entre Mathieu Guillet et ses enfants, 2 janvier 1655, notaire Jamon, 3 E 3312.

29. ADCM, inventaire des meubles et papiers de défunt Solon Baudoin, 29 avril 1673, notaire Lambert, 3 E 3324. 
Guillet, reçut entre autres «une maison et un jardin de 200 ceps à planter au village et dizaine du Morinand ${ }^{30}$ ».

Privilèges et intensification de la culture de la vigne ayant ainsi pu déterminer l'enracinement et l'attachement des Martinais à leur île notamment dans les familles d'immigrants qui possédaient, pour la plupart, des pièces de vigne, comme le montrent aussi les dotations d'enfants - il convient de s'interroger sur la taille de leurs exploitations viticoles et, quand les sources le permettent, sur leur degré d'aisance économique.

\section{Exploitation viticole et aisance des familles selon les apports au mariage}

D’après Sylvie Dépatie, plus encore que le degré d'égalité ou d’inégalité dans le mode de dévolution des biens, c'est en fait la taille du patrimoine familial qui détermine la réussite du processus de transmission et donc le sort de la génération suivante ${ }^{31}$. Il apparaît donc essentiel de connaître les biens dont disposaient les familles en vue de l'établissement de leur progéniture, car cela peut permettre de déterminer si les futurs immigrants disposaient ou non d'une place réelle à l'intérieur de la communauté et dans quelle mesure ils se trouvaient confrontés à un phénomène d'exclusion.

Les contrats de mariage martinais précisent généralement les apports à la nouvelle communauté et peuvent ainsi témoigner, même de façon sommaire et imparfaite, du niveau de fortune de la population concernée en l'absence d'inventaires après décès ${ }^{32}$. Comme ces apports sont souvent mentionnés non seulement en espèces mais également en pièces de vignes, on a dû calculer la valeur des pièces suivant leur superficie en leur attribuant la moyenne de 800 livres par quartier de vigne. Cette moyenne a été établie suivant un contrat de partage précisant la valeur des parcelles entre 600 et 1000 livres par quartier de vigne ${ }^{33}$, les parcelles étant considérées comme plus ou moins avantageuses selon leur situation et donc la qualité du sol où elles se trouvaient. Si l'on estime, avec Jean-Pierre

30. ADCM, contrat de partage entre Mathieu Guillet et ses enfants, 2 janvier 1655, notaire Jamon, $3 \mathrm{E} 3312$. Une dizaine correspondait à une forme de division de quartier dans certaines villes ou bourgs sous l'Ancien Régime.

31. Sylvie Dépatie, «La transmission du patrimoine dans les terroirs en expansion : un exemple canadien au xviII ${ }^{\mathrm{e}}$ siècle ", Revue d'histoire de l'Amérique française, 44,2 (automne 1990) : 171-198.

32. Louis Lavallée, "Les archives notariales et l'histoire sociale de la Nouvelle-France», Revue d'histoire de l'Amérique française, 28,3 (décembre 1974) : 393-394.

33. ADCM, contrat de partage des biens de feu Natanael Bernard, 24 mars 1674, notaire Lambert, 3 E 3323. 
TABLEAU 5

\section{Dotation des communautés futures selon les contrats de mariage se rapportant aux familles des immigrants $(n=26)$ *}

\begin{tabular}{l|c|c}
\hline & Dotation des femmes (\%) & Dotation des hommes (\%) \\
\hline Plus de 400 livres & 52 & 26 \\
De 100 à 400 livres & 15 & 26 \\
Droits successifs & 26 & 7 \\
Moins de 100 livres & 7 & 4 \\
Aucun apport précisé & 0 & 37 \\
\hline Total & 100 & 100 \\
\hline
\end{tabular}

* Il s'agit des contrats de mariage de l'ensemble des ascendants et collatéraux directs des immigrants.

Poussou, qu'il y a pauvreté rurale lorsque l'apport est inférieur à 100 livres et relative aisance lorsque les familles dotent leurs enfants de plus de 400 livres $^{34}$, le calcul en espèces des dots peut permettre une première approche du niveau de fortune des familles des immigrants (tableau 5).

Selon ces critères, nous serions en présence d'un milieu généralement assez aisé, du moins au-dessus du niveau de pauvreté, même si plusieurs contrats ne sont pas très explicites concernant la valeur des droits mobiliers et immobiliers dont disposaient les futurs conjoints. Plus de $85 \%$ des futures communautés jouissaient d'apports combinés de plus de 100 livres.

Ce milieu ne semble pas non plus représenter une société très contrastée, dans la mesure où le couple le mieux doté apportait 6000 livres et le plus misérable juste "un lit garny ${ }^{35}$ ». Ce qui est loin d'être en rapport avec le type de société attribué par René Plessix au Maine et au Perche de la seconde moitié du XviII ${ }^{\mathrm{e}}$ siècle, où l'apport le plus important était 16000 fois supérieur au plus faible ${ }^{36}$.

Les familles d'immigrants ne paraissent donc pas pour la plupart sans ressources, ce qui corrobore l'hypothèse avancée par Jean-Pierre Poussou,

34. Jean-Pierre Poussou, Bordeaux et le Sud-Ouest au XvIII ${ }^{e}$ siècle. Croissance économique et attraction urbaine (Paris, Éditions de l'École des hautes études en sciences sociales et J. Touzot, 1983), 301-320.

35. C'est-à-dire : un châlit ou bois de lit, une paillasse, un lit de plume, un traversin, deux draps et une couverture.

36. René Plessix, «Les tables de contrats de mariage, source pour une histoire sociale du Maine et du Perche dans la seconde moitié du xviII ${ }^{\mathrm{e}}$ siècle», dans Joseph Goy et Jean-Pierre Wallot, dir., Évolution et éclatement du monde rural : structures, fonctionnement et évolution différentielle des sociétés rurales françaises et québécoises, $\mathrm{XVII}^{e}$-XX ${ }^{e}$ siècles, op. cit., 286-295. 
selon qui il faut un minimum de liberté financière pour tendre à l'émigration $^{37}$, ainsi que celle de Leslie Choquette, qui a expliqué, dans le mouvement colonisateur, l'importance variable des diverses régions du Poitou par les différentes structures de propriété de ces pays. D’après elle, les bocages, qui couvraient la plus grande partie du Bas-Poitou, autour de Luçon et de Fontenay-le-Comte, étaient principalement exploités par des métayers qui tendaient à s'enliser dans le cercle vicieux de la pauvreté, de l'endettement et de l'instabilité géographique (micro-mobilité). Moins de colons sont donc partis des bocages que des plaines céréalières, entre Luçon et Niort, où les paysans étaient propriétaires de leurs champs. Même corrélation positive entre propriété et émigration dans les régions du Nord-Ouest ou du Centre-Ouest : les départs touchaient surtout les bénéficiaires du capitalisme, et non pas ceux qui se trouvaient en situation de dépendance vis-à-vis de l'ancien ou du nouveau système.

Les bases sociales et économiques de l'émigration [...] étaient les mêmes, peu importe la distance qui la séparait de l'Atlantique. Les régions qui ont participé au processus de modernisation économique et qui ont su en profiter ont donné plus d'émigrants que celles qui en sont restées à l'écart ou celles dans lesquelles les nouveaux mécanismes économiques ont généré plus de pauvreté que d'occasions d'affaires ${ }^{38}$.

La mesure de la superficie du patrimoine partagé après décès ou donation apporte également des précisions sur le niveau d'aisance des familles des futurs candidats à l'émigration. Globalement, selon Marcel Lachiver, une terre d'une superficie inférieure à un hectare conduisait pratiquement à la misère, cette surface étant trop réduite pour être en mesure de faire vivre une famille ${ }^{39}$. Jean-Pierre Poussou estime pour sa part qu'un quart d'hectare suffisait à un vigneron du Sud-Ouest pour nourrir une famille de cinq personnes grâce à la vente de son vin. Dans cette partie de l'île de Ré où la monoculture de la vigne était la règle, on peut en effet supposer que moins d'un hectare, c'est-à-dire la superficie moyenne observée, pouvait assurer la survie d'une famille. La plus grande des exploitations observées dépassait à peine trois hectares, soit moins que ce que Lachiver considère comme un gros vignoble, avec quatre à cinq hectares.

37. Jean-Pierre Poussou, "Les chemins de la migration", dans Yves Landry, John A. Dickinson, Suzy Pasleau et Claude Desama, Les chemins de la migration en Belgique et au Québec, $X V I I-X X^{e}$ siècles (Beauport/Louvain-la-Neuve, MNH/Academia, 1995), 9-20.

38. Leslie Choquette, op. cit., 66. Voir aussi 73.

39. Marcel Lachiver, Vins, vignes et vignerons. Histoire du vignoble français (Paris, Fayard, 1988), 245. 
En l'absence de grands domaines, il était fréquent de compléter son occupation principale par un travail annexe, à titre de vigneron gagé ou artisan par exemple, comme Mathieu Guillet et Jean Jalais, pères des émigrants Mathieu Guillet et Marie Jalais, qui alliaient les professions de laboureur et tonnelier. Les familles pouvaient également conjuguer le métier de vigneron avec celui de marchand de vin, comme Solon Baudoin, sieur des Marattes, le père de Jacques, qui possédait une propriété au lieudit des Marattes, dans la paroisse de Saint-Martin, où il disposait de plusieurs celliers avec tout le matériel pour faire le vin, comme un «treuil turquois et sa foullonoire», "une grande table de deux planches de sap et deux bancs de même grandeur, le tout servant à faire manger les vendangeurs» et "soixante-deux barriques de vin doux frelaté trois fois ${ }^{40}$ ». Cette combinaison de deux métiers permettait aux familles d'augmenter leurs revenus et de doter leur descendance en espèces plutôt qu'en terre, vu la taille réduite des exploitations, évitant ainsi de les morceler.

Ce mode de reproduction sociale implique également que si la communauté familiale devait allier différentes activités pour survivre, les parcelles héritées ne pouvaient évidemment suffire à l'établissement des enfants ni même à la formation d'une nouvelle exploitation. Les descendants étaient donc obligés de disposer aussi de revenus annexes pour survivre, surtout si le marché foncier était saturé comme c'était le cas sur cette île de 110 kilomètres carrés ${ }^{41}$. Mais un tel expédient n'était pas forcément un signe de pauvreté, bien au contraire, l'essor du négoce - en certaines années les vignes rhétaises pouvaient produire jusqu'à 55000 tonneaux ${ }^{42}$ — et la propriété d'au moins quelques parcelles de vigne assurant plutôt une relative aisance de la plupart des familles. Confirmant cet état de fait, les contemporains considéraient que, malgré des exploitations de taille réduite, cette population n'était nullement misérable. L'intendant Bégon affirma qu'il n'y avait pas un mendiant dans l'île, en dépit d'une période économique difficile ${ }^{43}$.

40. ADCM, inventaire des meubles et papiers de défunt Solon Baudoin, 29 avril 1676, notaire Lambert, 3 E 3324.

41. L'île de Ré comptait près de 20000 habitants au début du xviII ${ }^{\mathrm{e}}$ siècle, correspondant à une densité de population de 150 à 200 habitants au $\mathrm{km}^{2}$, par rapport à une densité moyenne en France de 30 habitants au km² en 1750. Louis Perouas, Le diocèse de La Rochelle de 1648 à 1724. Sociologie et pastorale (Paris, SEVPEN, 1964), 106-107, citant Claude Masse, Mémoire géographique du Bas-Poitou, pais d'Aunis et Saintonge (1712), $1: 79$.

42. Marcel Lachiver, op. cit., 266, citant Claude Masse, Mémoire géographique du Bas-Poitou, pais d'Aunis et Saintonge (1712).

43. Michel Bégon, «Mémoire sur la généralité de La Rochelle [1698]», Archives historiques de la Saintonge et de l'Aunis, 2 (1875): 54. 
Claude Masse renchérit un peu plus tard en soutenant que «le peuple y est très aisé ${ }^{44}$ ».

L’omniprésence de la vigne dans la majorité des transactions notariées, qu'il s'agisse de ventes et de constitutions de rente, ou encore de partages et de contrats de mariage, n'était pas sans créer un rapport spécifique à la terre qui poussait encore davantage cette population à la sédentarité. Comme le souligne Marcel Lachiver, «de tous les groupes sociaux qui ont été étudiés, ce sont les vignerons qui ont la plus faible mobilité ${ }^{45}$ ». Ainsi, ils se trouvaient fortement attachés à la terre de leurs parents qui euxmêmes s'étaient souvent établis sur le territoire où ils étaient nés. En outre, comme c'était de la production de ces parcelles dont dépendait la survie de la communauté, dans un pays agricole où les revenus provenaient essentiellement de la terre, il s'agissait de tout mettre en œuvre pour que les biens fonciers ne sortent pas de la famille : l'attachement au pays possédait de solides raisons foncières. Si le garçon restait sur place, c'est qu'il avait été pourvu, comme sa future épouse, d'un apport en biens fonciers provenant du patrimoine familial et constitué de vignes situées dans la paroisse de Saint-Martin ou du moins dans l'île de Ré. La nouvelle communauté se trouvait donc dans l'obligation de rester dans la paroisse pour cultiver les terres reçues au mariage. Quitter le lieu d'origine aurait signifié recourir au marché foncier pour acquérir une terre. Il apparaissait plus simple de poursuivre l'exploitation familiale dans une situation de saturation de ce marché.

Ainsi, le vigneron se révélait un homme plus souvent propriétaire de sa terre que les autres paysans et donc plus enraciné dans le sol qu'il possédait. Son attachement résidait également dans le fait que la viticulture n'était pas une culture comme les autres. Il s'agissait d'une culture pérenne, pouvant vivre plus longtemps que l'homme, et le vigneron savait par exemple que telle vigne avait été plantée par son grand-père et à quelle date précise. Tous les insulaires, et les moins aisés plus que les autres parce qu'il s'agissait de leur seul bien, étaient attachés aux vignes et aux champs qu'avaient labourés leurs ancêtres et où ils travaillaient depuis leur enfance ${ }^{46}$.

44. Louis Perouas, op. cit., 106-107, citant Claude Masse, Mémoire géographique du Bas-Poitou, pais d'Aunis et Saintonge (1712), $1: 79$.

45. Marcel Lachiver, op. cit., 238.

46. Ibid., 241. 


\section{STRATÉGIES FAMILIALES ET TENTATIVES DE PRÉSERVATION DU PATRIMOINE}

Dans un tel contexte, il est indéniable qu'en l'absence de terre à cultiver ou de possibilités de mariage, l'avenir pouvait paraître incertain à de jeunes Rhétais. À défaut de pratiquer un autre métier sur place, il fallait alors quitter l'île soit en direction du continent dans l'arrière-pays aunisien, soit outre-Atlantique vers les colonies.

Cet aspect de la question aborde les causes de la migration dans la perspective que la saturation démographique d'un terroir pouvait provoquer le blocage des stratégies de transmission du patrimoine familial, l'émigration vers d'autres zones de peuplement étant le résultat de ce blocage ${ }^{47}$. Suivant cette piste de recherche, il nous est apparu nécessaire, dans un premier temps, de connaitre le mode de transmission des biens dans l'île de Ré pour vraiment cerner le degré d'égalité entre les héritiers et ainsi l'orientation du système de reproduction sociale.

\section{La coutume de Paris et le partage égalitaire}

L'île de Ré dépendait certes de la coutume de La Rochelle et du pays d'Aunis, mais celle-ci suivait communément la coutume de Paris, qui réglait les successions selon un mode de partage égalitaire. Contrairement à d'autres régions où le partage égalitaire pouvait être plus ou moins bien observé, il ressort de l'étude des contrats de partage des familles des émigrants de la paroisse de Saint-Martin entre 1655 et 1689 que l'égalitarisme entre les enfants était un principe suivi pratiquement à la lettre.

Rappelons que les modes de dévolution égalitaire prévoyaient l'exclusion des enfants précédemment dotés avec une possibilité d'option : ils pouvaient s'en tenir aux avantages acquis ou les reporter à la masse de la succession, laquelle était ensuite partagée par moitié entre le conjoint survivant et les héritiers ${ }^{48}$. Suivant ces principes, les descendants étaient appelés en premier

47. Gérard Bouchard, «La reproduction familiale en terroirs neufs. Comparaison sur des données québécoises et françaises», Annales ESC, 48,2 (mars-avril 1993) : 421-451. Gérard Bouchard, John A. Dickinson et Joseph Goy, dir., Les exclus de la terre en France et au Québec, XVII ${ }^{e}$-XX ${ }^{e}$ siècles : la reproduction familiale dans la différence (Sillery, Septentrion, 1998), 336 p. Pour un exemple canadien d'une telle situation dans la première moitié du xviII ${ }^{\mathrm{e}}$ siècle, voir Yves Landry et Réal Bates, «Population et reproduction sociale à l'île d'Orléans aux xvII et XviII siècles », Revue d'histoire de l'Amérique française, 45,3 (hiver 1992) : 403-413.

48. Dominique Joulia, «Pratiques successorales en milieu rural 1795-1820 : étude comparative de cas France de l'Ouest - Québec», dans Joseph Goy et Jean-Pierre Wallot, dir., Société rurale dans la France de l'Ouest et au Québec (XVII ${ }^{e}$-XX $X^{e}$ siècles) (Montréal/Paris, Université de Montréal/École des hautes études en sciences sociales, 1981), 93-140. 
lieu. Les enfants, dans le cadre légal de la reproduction sociale, recueillaient les biens de leurs parents suivant un principe de stricte égalité, sans droit d'aînesse ni privilège de masculinité. Après estimation des biens meubles et immeubles, des lots étaient constitués, chacun d'une valeur égale, et tirés au sort entre les héritiers. Quand une égalité parfaite était impossible, les titulaires des lots de valeur supérieure devaient payer la différence, calculée en espèces, aux héritiers des autres lots. Ainsi, les biens de Nicolas Dessandy, le grand-père maternel de l'émigrante Marie Jalais, paraissent avoir été partagés équitablement entre ses deux enfants, Michel, «tixier en toile» dans le bourg de La Flotte comme son père, et Bertholomée, épouse de Jean Jalais résidant à Saint-Martin : le 2 novembre 1661, Bertholomée reçut deux pièces de vigne de 1800 et 810 ceps situées «d'un bout au grand chemin qui conduit de La Flotte à Sainte-Marie» et Michel, une pièce de vigne de 1400 ceps ainsi que «la moitié, avec les héritiers de Simone Ferneau, à qui l'autre moitié appartient, d'une maison à fest, plancher et couverte de tuiles, située à La Flotte, avec la moitié d'un jardin proche de la maison contenant tout le jardin, $48 \operatorname{ceps}^{49}$ ». Les deux héritiers se sont également partagé les meubles et effets. Cet exemple montre l'extrême souci d'égalité concernant le partage des biens pour qu'aucun héritier ne soit lésé.

Cependant, on le verra plus loin, la réalité pouvait nuancer les prescriptions de la coutume de Paris. Comme les biens fonciers se transmettaient dans des familles établies dans l'île de Ré depuis souvent plusieurs générations, ils faisaient l'objet de liens relativement forts et suscitaient une attention particulière à l'intérieur des familles.

\section{Les possibilités d'exclusion dans le milieu rural rhétais}

Ce système en terroir plein ne semble pas permettre l'établissement de tous les enfants, qu'ils fussent exclus du mariage ou exclus de l'exploitation dans un mode de reproduction sociale à l'identique. Le concept d'exclusion est alors déterminé par le fait, pour un fils ou une fille de paysan, de ne pas accéder à la propriété ou à la gestion de la terre, mais cette définition sera encore une fois à nuancer.

Devant les lacunes des registres paroissiaux et des minutiers auxquelles nous avons été exposés pour reconstituer le patrimoine familial et observer son devenir, il est difficile de généraliser les résultats obtenus à l'ensemble des stratégies adoptées et aux diverses formes prises par la repro-

49. ADCM, partage des biens de Nicolas Dessandy, 2 novembre 1661, notaire Lambert, 3 E 3319. 
duction sociale au sein des familles. Tout au plus, pouvons-nous évoquer des hypothèses et faire état de quelques cas pour illustrer notre propos.

Tout d'abord, il paraît utile de souligner, même en l'absence d'analyse démographique rigoureuse, que ces familles enracinées dans l'île depuis plusieurs générations jouissaient souvent d'une espérance de vie relativement longue. Ainsi il n'était pas rare que des enfants se marient bien avant le décès ou la donation de leurs parents, comme ce fut le cas de Maurice Guillet, le grand-père de Mathieu Guillet, qui épousa Marie Moynard le 10 février $1642^{50}$, treize ans avant la donation de son père pour cause d'incapacité à gérer lui-même ses biens ${ }^{51}$. Même âgés, les parents tentaient, semble-t-il, de conserver leur indépendance le plus longtemps possible et n'abandonnaient leurs biens et ne prenaient pension chez leurs enfants qu'en dernière extrémité.

Ce retard pouvait induire un blocage de la reproduction sociale même si des dons avaient été effectués. En effet, s'ils avaient été le fait du grandpère, les parents en profitaient, mais les enfants devaient encore attendre le décès ou la donation des parents pour s'établir et ainsi avoir accès à une partie du patrimoine familial. Dans l'attente d'une succession, ou parfois dans la foulée d'un héritage, certains émigrants pouvaient décider de s'expatrier en vue de trouver un milieu plus propice à leurs ambitions. Par exemple, Louis Marchand et son épouse Françoise Morineau vendirent quatre mois après le décès d'une tante une parcelle de vigne provenant de sa succession pour la somme de 170 livres $^{52}$, transportèrent quelques années plus tard une rente foncière de 26 livres annuelles et enfin s'embarquèrent pour la colonie. Ces ventes leur avaient peut-être permis d'acquérir suffisamment de fonds pour partir avec leurs quatre enfants et rejoindre leur fils aîné préalablement émigré au Canada.

Nous connaissons trois cas où le départ est intervenu après le remariage d'un des parents. Par exemple, Anne Leblanc se maria en Nouvelle-France seulement deux ans après le remariage de son père ${ }^{53}$, celui-ci intervenant un an après le décès de son épouse ${ }^{54}$. On peut penser que le père de l'immigrante, au moment de son second mariage, a octroyé à sa fille les droits

50. ADCM, contrat de mariage entre Maurice Guillet et Marie Moynard, 10 février 1642, notaire Jamon, 3 E 3312.

51. ADCM, partage Guillet, 2 janvier 1655, notaire Jamon, 3 E 3312.

52. ADCM, vente de Louis Marchand et de Françoise Morineau à Gabriel Sourisseau, 21 mars 1658, notaire Jamon, 3 E 3313.

53. ADCM, mariage de Nicolas Leblanc et Suzanne Prevost, 29 juillet 1670, registres paroissiaux de Saint-Martin.

54. ADCM, sépulture d'Anne Gauthier, 29 mars 1669, registres paroissiaux de Saint-Martin. 
successifs relatifs au décès de sa précédente épouse, lesquels formaient son apportionnement. Anne Leblanc profita finalement des avantages consentis aux Filles du roi pour partir au Canada.

Rappelons que, lors de son remariage, le parent survivant apportionnait ses enfants d'une somme donnée, qui était variable selon son degré d'aisance, en compensation de leurs droits par rapport au parent décédé, ce qui pouvait les priver de tout droit lié à cette succession. Dans ce cas, le survivant gardait en retour la jouissance des biens du conjoint décédé et les enfants ne prenaient possession de ces biens qu'à leur majorité ou à leur mariage. Par exemple, Pierre Rocand et Jean Gautron - qui ne paraissent pas s'être embarqués comme engagés — ont peut-être attendu leur majorité et leurs droits pour s'expatrier, le remariage respectivement de leur mère et de leur père intervenant alors qu'ils étaient encore enfants. Le partage des biens pouvait donc avoir lieu immédiatement après le décès, mais il pouvait aussi n'intervenir qu'à la majorité d'un des héritiers ou au remariage du conjoint survivant. Ajoutons que s'il n'y avait pas de remariage, l'indivision pouvait persister parfois jusqu'au décès du conjoint survivant ${ }^{55}$. En cas inverse, la situation pouvait se compliquer encore davantage si la nouvelle communauté engendrait d'autres enfants, comme dans l'exemple de Jacques Baudoin qui, outre ses trois frères et sœurs, se trouva avec au moins quatre autres enfants, dont trois garçons, issus du second mariage de son père.

La coutume de Paris donnait donc tout de même un pouvoir important à la communauté des parents : celui de favoriser ou non un héritier et d'aider plus ou moins la nouvelle communauté d'un enfant par le biais des dots, avancements d'hoirie et partages du vivant des parents ${ }^{56}$.

Observons que les émigrants de Saint-Martin n'étaient jamais les aînés et occupaient souvent un rang de naissance lointain dans la famille. Au mieux, l'émigrant était le second des garçons survivants, comme Jacques Baudoin, et au pire dans une famille comprenant huit enfants il était le quatrième fils, à l'instar d'André Mignier. Les règles déterminant aussi bien le candidat à l'émigration que le successeur restent obscures, car elles faisaient intégralement partie des stratégies familiales. Le successeur pouvait être un cousin comme dans le cas d'André Boissonnière, cousin de

55. Sylvie Dépatie, loc. cit., 177-178.

56. Thiphaine Barthelemy de Saizieu, «Les alliances matrimoniales à Neuville à la fin du XVIII ${ }^{\mathrm{e}}$ siècle», dans Joseph Goy et Jean-Pierre Wallot, dir., Évolution et éclatement du monde rural : structures, fonctionnement et évolution différentielle des sociétés rurales françaises et québécoises, $\mathrm{XVII}^{e}$-XX $\mathrm{X}^{e}$ siècles, op. cit., 315-323. 
Gilles Asselin, ce dernier étant le père de Françoise qui se maria à Québec en 1669. André racheta les parties de la maison de leur grand-mère Marie Turbé située dizaine du Four à Saint-Martin, éparpillées entre ses différents héritiers ${ }^{57}$. Mais il pouvait également s'agir d'un cadet ou du seul fils survivant. Ainsi, Jean Jalais et Bertholomée Dessandy, les parents de Marie, vendirent à leur unique fils, Nicolas, une maison pourvue d'un jardin, sise dizaine des Forges à Saint-Martin, pour la somme de 60 livres que le père lui devait ${ }^{58}$. Cela permit ainsi au frère de l'immigrante de s'établir tout en délivrant ses parents de quelques dettes.

Nous croyons à l'instar de Bernard Derouet dans le Bourbonnais que, plutôt qu'une cause de l'émigration, l'exclusion de la transmission des biens immobiliers était une conséquence du départ de l'émigrant.

Seuls héritent pleinement du patrimoine ceux des enfants dont le destin prévu est de rester intégrés à leur communauté de naissance, alors que ceux qui doivent partir dans une autre communauté, ou partir au loin, sont au contraire "appanés», c'est-à-dire qu' ils reçoivent alors une petite dot mobilière pour tout héritage à venir $[\ldots]$. La motivation profonde de ce mode d'héritage est de chercher à empêcher que des personnes extérieures à la communauté aient des droits sur son assise foncière; elle est de faire coïncider résidence sur le domaine et propriété sur ce même domaine ${ }^{59}$.

Ce phénomène explique le silence observé à l'égard des émigrants dans les partages postérieurs au départ qu'il nous a été donné d'étudier. Tout au plus leurs familles étaient-elles informées du moment de leur décès. Ainsi Isaac Dodin, tonnelier à Saint-Martin, émigra avec sa fille Hélène et apparut en Nouvelle-France dès 1663. Il laissa dans le bourg Anne Jarnet, sa femme, et au moins deux de leurs enfants, Marthe et Jacques. Ce dernier, charpentier de marine à Saint-Martin, épousa Rachel Guilbaud selon un contrat de mariage établi le 23 mai 1683 où il précisait que son père était décédé60, ce qui était le cas puisque Isaac avait été enterré à Montréal le 7 avril 1671.

Comme nous l'avons vu, la communauté était liée à l'exploitation qui conditionnait sa survie. En ce sens, si l'héritier ne résidait pas dans la pa-

57. ADCM, vente de Gilles Asselin à André Boissonnière, 4 février 1662, notaire Jamon, 3 E 3313.

58. ADCM, vente de Jean Jalais à Nicolas Jalais, 14 septembre 1662, notaire Bouriau, 3 E 3487.

59. Bernard Derouet, «Pratiques successorales et rapport à la terre : les sociétés paysannes d'Ancien Régime", Annales ESC, 44,1 (janvier-février 1989) : 192. Voir aussi Bernard Derouet, "Territoire et parenté, pour une mise en perspective de la communauté rurale et des formes de reproduction sociale", Annales d'histoire et sciences sociales, 3 (mai-juin 1995) : 645-686.

60. ADCM, contrat de mariage entre Jacques Dodin et Rachel Guilbaud, 23 mai 1683, notaire Jamon, 3E 3315 
roisse, il pouvait être privé de ses droits concernant le patrimoine au bénéfice d'une communauté établie sur place, et cela malgré un partage égalitaire strict. Il perdait ainsi ses droits parce qu'il avait été exclu ou parce qu'il s'était exclu lui-même par son départ. Il ne restait plus à l'immigrant qu'à tenter de faire valoir ses intérêts, en rentrant en métropole ou en s'y faisant représenter, ce qu'illustrent bien certaines histoires présentées ailleurs ${ }^{61}$.

Peut-on imaginer des cas d'exclusion par exhérédation qui auraient provoqué l'émigration d'un enfant? Il ne pourrait s'agir que d'un phénomène rare, car les parents ne pouvaient pas écarter purement et simplement leurs enfants de la succession. Certes la législation prévoyait certaines causes permettant de le faire, lesquelles se répartissent en deux grandes catégories, soit «l'ingratitude des enfants envers leurs parents» ou encore "les comportements non tolérés de la part du descendant considéré, [c'està-dire] le dérèglement de mours ${ }^{62}$ ", mais ces conduites sont bien difficiles à confirmer ou à infirmer. Cependant le cas de Jacques Baudoin peut semer le doute en ce qui concerne la rareté du phénomène puisque, fils cadet d'un important négociant et propriétaire de Saint-Martin, il partit pour la Nouvelle-France en tant qu'engagé et se trouva totalement écarté de nombreuses successions, alors que d'autres membres de sa famille proche, émigrés aux Antilles, prirent tout de même part à l'héritage.

Malgré la diversité des situations préalables au départ, une réalité paraît commune à tous les immigrants observés : aucun, même héritier, n'était successeur de l'exploitation principale. La pluralité des héritiers, le décès tardif des parents, le remariage du conjoint survivant, les stratégies matrimoniales, la saturation du marché foncier, tous ces éléments pouvaient conférer à l'immigrant un statut d'exclu de l'exploitation s'il ne trouvait pas à se marier sur place et à ainsi accéder à la terre. Ajoutés à la position géographique et à l'activité économique du port de Saint-Martin, en face de celui de La Rochelle, ces facteurs ne sont pas sans avoir facilité le départ des Martinais.

61. Voir l'exemple du soldat versaillais François-Thomas Giroux, cité dans Yves Landry, «Les Français passés au Canada avant 1760 : le regard de l'émigrant", loc. cit., qui montre que des immigrants restaient bien attachés à leur patrimoine familial, malgré l'éloignement et les années passées au loin, surtout si les biens hérités étaient de quelque conséquence.

62. Geneviève Postolec, «L'exclusion de la succession par exhérédation ou par substitution au Canada aux xvII et XviII ${ }^{\mathrm{e}}$ siècles", dans Gérard Bouchard, John A. Dickinson et Joseph Goy, dir., Les exclus de la terre en France et au Québec, $\mathrm{XVII}^{\mathrm{e}}{ }^{-} \mathrm{XX} \mathrm{X}^{\mathrm{e}}$ siècles : la reproduction familiale dans la différence, op. cit., 36-37. 


\section{L'INFLUENCE D’UNE ACTIVITÉ PORTUAIRE EN EXPANSION}

L'influence de zones d'activités économiques florissantes tournées vers l'Atlantique à proximité du lieu d'origine d'émigrants en Nouvelle-France n'a pas été bien étudiée, bien qu'à la suite de Gabriel Debien ${ }^{63}$ beaucoup aient convenu de son importance. Nous ne prétendons pas remédier totalement à cette lacune, même si les réseaux économiques unissant les deux ports de Saint-Martin et de La Rochelle, leurs trafics maritimes outreAtlantique, ainsi que l'établissement de Martinais aux Antilles faisaient étroitement partie de l'environnement dans lequel baignaient les futurs émigrants.

Il convient de s'intéresser également au climat religieux de Saint-Martin marqué par la proximité de la ville de La Rochelle. En effet, c'est par cette dernière que sont apparues et se sont développées les idées de la Réforme largement répandues à Saint-Martin. De ce fait, on comprendra plus aisément la présence de quelques huguenots dans le groupe des Martinais expatriés, comme Jacques Baudoin, Isaac et Hélène Dodin, Barbe Ménard et Nicolas Geoffroy. Déjà évoqué plus haut, ce thème longtemps controversé a été souvent négligé puisque, selon le roi, les colonies ne devaient être peuplées que de «naturels François catholiques».

\section{Les relations entre les ports de La Rochelle et de Saint-Martin}

L'influence et la proximité de La Rochelle, un des principaux ports de commerce de la façade atlantique, ne sont pas étrangères au développement de Saint-Martin. Rappelons que La Rochelle, née au XII siècle, a acquis une grande importance économique avec l’importation du blé, du vin et du sel d'Aunis. Le havre est devenu, dès le xiv ${ }^{\mathrm{e}}$ siècle, un des principaux ports de commerce français et, vers la fin du $\mathrm{xvI}^{\mathrm{e}}$ siècle, un centre actif du commerce avec l'Europe du Nord, l'Espagne et les mers lointaines. Cela grâce à des " "franchises exceptionnelles", peu à peu acquises depuis la guerre de Cent Ans et maintenues âprement ${ }^{64}{ }^{4}$ à l'instar de la baronnie de Ré.

Le commerce des vins entre l'île de Ré et La Rochelle semble florissant dès le XIII ${ }^{\mathrm{e}}$ siècle. Selon Étienne Trocmé et Marcel Delafosse, à la fin du $\mathrm{xvI}^{\mathrm{e}}$ et au début du $\mathrm{xvII}^{\mathrm{e}}$ siècle, La Rochelle entretenait non seulement des

63. Gabriel Debien, «Engagés pour le Canada au XviI ${ }^{\mathrm{e}}$ siècle vus de La Rochelle», Revue d'histoire de l'Amérique française, 6,2 (mars 1952) : 177-233, et 6,3 (décembre 1952) : 374-407.

64. Élisabeth Forlacroix, "La famille protestante à La Rochelle avant la Révocation», dans Anne Blanchard, Henri Michel et Élie Pelaquier, dir., Famille et familles dans la France méridionale à l'époque moderne (Montpellier, Université Paul-Valéry Montpellier III, 1992), 68. 
relations avec son vaste arrière-pays mais également, grâce à ses privilèges douaniers, avec l'Angleterre et les Provinces Unies, le Portugal et l'Espagne du Sud, et enfin le Canada dont "quelques Rochelais audacieux tiraient, malgré de grandes difficultés, de riches cargaisons de fourrures ${ }^{65}$ ». Ces rapports commerciaux étaient importants et fréquents, les marchands étrangers venant aussi chercher du vin, de l'eau-de-vie et du sel. Ils concluaient leurs affaires à La Rochelle et chargeaient ensuite les produits dans des ports voisins comme Saint-Martin. À ce titre, l'île de Ré occupait une position satellite par rapport à La Rochelle, toutes deux étant filles du grand commerce européen ${ }^{66}$.

Le début du trafic entre La Rochelle et la colonie des Antilles date de 1636, avec la mise en place d'un commerce régulier qui, d'abord, n'occupait que quelques navires par an ${ }^{67}$. L'augmentation considérable du nombre des départs, notamment entre 1660 et 1686, entraîna la constitution d'une flotte affectée au voyage en direction des îles. Les quelque 4800 contrats d'engagement en direction des Antilles conservés pour la période 1620 à $1715^{68}$ font ainsi paraître le port de La Rochelle, avec celui de Dieppe, comme l'un des plus hauts lieux français du commerce colonial.

Le développement des relations commerciales de Saint-Martin avec La Rochelle, premier port d'embarquement pour les colonies et l'étranger, a donc familiarisé sa population avec les voyages au long cours et facilité sinon poussé une partie de sa population, à l'étroit sur cette terre, vers des horizons plus lointains, notamment les Antilles, qui ont vu débarquer des Martinais tout au long des $\mathrm{XVII}^{\mathrm{e}}$ et $\mathrm{XvIII}^{\mathrm{e}}$ siècles $^{69}$. Par exemple, Pierre Bruny, sieur de Baupin, marchand de Saint-Martin associé au sieur François de Thoumeau, maître de navire à La Tremblade, y envoya neuf hommes en février et avril 1642, puis sept autres en octobre $1642^{70}$.

65. Étienne Trocmé et Marcel Delafosse, Le commerce rochelais de la fin du XVI siècle au début du XVII ${ }^{e}$ (Paris, Armand Colin, 1952), 171.

66. Frédéric Grignon, La Rochelle : de la catastrophe au redressement. Population et comportements démographiques dans la première moitié du XVII eiècle (1629-1660), mémoire de maîtrise (histoire), Université de Poitiers, 1993, 160 p.

67. Marcel Delafosse, "La Rochelle et les îles au xvıI ${ }^{\mathrm{e}}$ siècle », Revue d'histoire des colonies, 36,3-4 (1949) : 240-243.

68. Gabriel Debien, Les engagés pour les Antilles (1634-1715) (Paris, 1952), $277 \mathrm{p}$.

69. Pierre Tardy, «Les Rétais et le nouveau monde. Saint-Domingue», Cahiers de la Mémoire [Groupement d'Études Rétaises, Revue d'art et tradition populaires d'archéologie et d'histoire], 48 (été 1992) : 1-24.

70. Gabriel Debien, Les engagés pour les Antilles (1634-1715), op. cit., citant les minutes Moreau, notaire à La Rochelle, le 31 octobre 1642, 37-38. 
Les marchands rhétais regroupés dans les deux ports principaux de l'île, Saint-Martin et La Flotte, étaient en relation étroite avec les marchands rochelais et souvent unis avec eux par des liens familiaux ${ }^{71}$. Par exemple, le marchand Jacques Baudoin, frère de l'émigrant du même nom, est né à Saint-Martin mais résidait à La Rochelle. Ses enfants, Jean-Charles et Marie Baudoin, sont nés également à La Rochelle et s'y sont mariés. Cette dernière y a épousé Louis Lepays, sieur de Bourjoly, avant de tenter l'aventure vers Saint-Domingue. Ainsi peut-on soupçonner une émigration martinaise en direction de La Rochelle, ou tout au moins un passage obligé dans ce port, premier lieu d'embarquement vers les colonies, pour faciliter l'expatriation.

\section{Développement du commerce de Saint-Martin et émigration : l'exemple des Antilles}

Le havre de Saint-Martin a été aménagé en 1597 mais jusqu'en 1685 ce n'était qu'un large chenal bordé de terre. Il fallut attendre 1672 pour que la marine royale s'intéressât au port de Saint-Martin et que Colbert de Terron ordonnât de débarrasser le port de ses épaves. Après la construction de l'enceinte fortifiée, on paracheva l'ouvrage en revêtant le havre de pierre de taille, ce qui évitait l'éboulement de la terre. On construisit également des cales et des quais sur le pourtour et le port subit un approfondissement, un agrandissement ainsi qu'un nettoyage complet du bassin de retenue. Ces travaux dotèrent la paroisse d'un port efficace, répondant aux besoins d'un trafic commercial croissant.

Si la plus grande activité commerciale du port de Saint-Martin se situe dans la première moitié du $\mathrm{xvIII}^{\mathrm{e}}$ siècle, le trafic était important dès le siècle précédent, avant même les travaux de restauration, du fait de la production locale abondante en vins, eaux-de-vie et sel, et de l'entreposage de marchandises étrangères résultant de l'absence de droits d'entrée dans l'île. Quelques chartes-parties retrouvées dans les minutiers des notaires martinais témoignent du commerce de Saint-Martin avec des pays étrangers et notamment les pays du Nord. Entre 1660 et 1668, 13 chartes-parties attestent un commerce en direction de pays comme l'Espagne, la Suède, la Norvège, l'Irlande, l'Angleterre et Terre-Neuve. Citons, à titre d'exemple, la frégate martinaise $\mathrm{La}$ croix d'or qui transporta vers le port suédois de Göteborg, en 1660, 25 tonneaux et barriques de vin et vinaigre, 14 barriques d'eau-de-vie et 28 barils de figues ${ }^{72}$. Vers Terre-Neuve, les

71. Étienne Trocmé et Marcel Delafosse, op. cit., 143.

72. ADCM, charte-partie entre Jean Leblanc et Pierre Mariau, 3 avril 1660, notaire Lambert 3 E 3319. 
navires faisaient route pour «faire la pescherie du poisson vert ${ }^{73}$ » ou " pour la pecsherie du poisson $\mathrm{seq}^{74}$ ».

Lieu de transit et d'entrepôt face au continent mais aussi siège de la contrebande qui en découlait ${ }^{75}$, l'île de Ré s'est trouvée, dès le $\mathrm{xvII}^{\mathrm{e}}$ siècle, trop exiguë pour le nombre et les ambitions de ses marchands dont la majorité résidait dans le bourg de Saint-Martin. Certains sont donc allés chercher fortune ailleurs, tout en conservant des liens avec leur milieu d'origine. La famille Baudoin, négociants et grands propriétaires de vignobles, marais salants et fiefs, en est un bon exemple. Le cas de Benoît Baudoin, oncle du futur émigrant Jacques Baudoin, permet ainsi de mieux cerner cette tradition migratoire familiale.

Benoît semble être né à Saint-Martin, mais il était domicilié à La Rochelle, comme l'indique son contrat de mariage passé devant le notaire rochelais Teuleron le 20 novembre 1645 . À cette occasion, il avantagea sa future épouse Louise Grignon de 6000 livres à charge de le suivre aux îles d'Amérique ${ }^{76}$. Le couple s'est apparemment installé dans l'île de la Martinique puisque les époux de Suzanne et Louise Baudoin, leurs filles établies "en ce bourg de saint-pierre ${ }^{77}$ ", y reçurent une procuration pour se rendre au bourg de Saint-Martin. Suzanne Baudoin avait épousé François Jarday, sieur des Marinières, et sa sœur Louise Baudoin était mariée à Nicolas Henry, sieur de Saint-Amour, tous deux habitants de SaintPierre, paroisse de la Martinique. L'acte de procuration a été établi en 1675 par Jean Gervays, «notaire de l'île de la martinique», pour le partage des biens de leur tante Louise Baudoin ${ }^{78}$, veuve de René Macquin, sieur de La Prée. Leur oncle Solon Baudoin eut d'ailleurs la jouissance des biens situés dans l'île de Ré et leur appartenant par le décès de leur père, Benoît Baudoin, jusqu'en $1675^{79}$.

Mais encore, Solon Baudoin, père de l'émigrant Jacques Baudoin, eut plusieurs petits-enfants déployant des activités antillaises centrées sur la

73. ADCM, charte-partie entre Simon Regreny, François Baudoin, Jean Richard et Pierre Resnier, 25 août 1668, notaire Lambert, 3 E 3322.

74. ADCM, charte-partie entre François Baudoin, Jacques Boutet et Jean Courtin, 20 mars 1677, notaire Lambert, 3 E 3324.

75. Pierre Tardy, loc. cit.

76. ADCM, contrat de mariage entre Benoît Baudoin et Louise Grignon, 20 novembre 1645, notaire Teuleron, 3E 1294.

77. ADCM, procuration de Louise Baudoin à Nicolas Henry, 20 juin 1675, notaire Lambert, 3 E 3323.

78. ADCM, partage Baudoin, 14 janvier 1673, notaire Lambert, 3 E 3323.

79. ADCM, arrêt de compte, quittance et cassation entre François Jarday, Nicolas Henry et Solon Baudoin, 9 septembre 1675, notaire Lambert, 3 E 3323. 
côte méridionale de Saint-Domingue, vers Jacmel. En effet, Jean-Charles Baudoin, fils de Jacques Baudoin et de Marie Massiot, le frère homonyme de l'immigrant en Nouvelle-France, résidait à Jacmel et y fit souche. En outre Marie Massiot avait plusieurs parents qui faisaient partie des directeurs de la compagnie formée en 1673 "pour le commerce du Sénégal», où il faut entendre la traite des noirs. Marie Massiot était elle-même, après «l'union» des compagnies d'Afrique en 1701 sous le nom de "Compagnie de l'Asiento", une des directrices de la nouvelle société ${ }^{80}$.

Rappelons également que Marie Baudoin, sœur aînée de Jean-Charles, mariée à La Rochelle en 1704 avec Louis Lepays, sieur de Bourjoly, est aussi allée vivre à la Grande Isle où est mort son mari en 1746 à Cayes du Fond.

Les exemples ne manquent donc pas dans la famille de Jacques Baudoin pour démontrer que Saint-Martin n'a pas été absente de l'émigration vers les colonies antillaises et que l'émigration vers la Nouvelle-France et les Îles pouvait coexister dans la même famille.

Un autre cas similaire est celui de François Bodaire, cousin germain de l'immigrante Marie Langlois, qui indiqua dans son testament rédigé en 1664 en faveur de sa mère Anne Langlois qu'il était «sur le point de s'embarquer pour faire voiage sur mer aux îles de la Guyane ${ }^{81}$ ", cette jeune colonie où la compagnie des Indes Occidentales envoya ses premiers engagés en septembre $1664^{82}$. Du fait de son absence ultérieure dans les sources martinaises, il semble ne pas être revenu à son lieu d'origine.

Si les deux ports de La Rochelle et de Saint-Martin apparaissent très liés d'un point de vue commercial, et que le négoce qu'ils entretenaient avec l'étranger a pu faciliter l'émigration outre-Atlantique, les affinités religieuses de leurs habitants ont pu aussi contribuer au même effet.

\section{Présence protestante}

L’origine huguenote des émigrants a été établie selon leur présence dans les registres du culte protestant martinais et rochelais, ainsi que d'après la mention "selon la religion prétendue réformée» présente dans les contrats de mariage de leur parentèle. Les traces d'abjuration en Nouvelle-France ont également été utiles. Cependant, comme les inévitables lacunes pré-

80. ADCM, contrat de mariage entre Jacques Baudoin et Marie Massiot, 12 avril 1687, notaire Soulard, 3E 1808. Gabriel Debien, Les engagés pour les Antilles (1634-1715), op. cit., 155-156.

81. ADCM, testament de François Bodaire, 6 février 1664, notaire Lambert, 3 E 3320.

82. Gabriel Debien, Les engagés pour les Antilles (1634-1715), op. cit., 152. 
sentes dans les sources notariales et paroissiales ne permettent pas d'être exhaustif quant à l'origine religieuse des émigrants, il ne peut être fait état ici que des protestants avérés.

La proportion d'huguenots parmi les émigrants martinais du $\mathrm{xvII}^{\mathrm{e}}$ siècle vers le Canada atteint un minimum de $13,5 \%$. Ce chiffre n'étant pas négligeable, il convient de s'interroger sur le contexte religieux de la paroisse ainsi que sur les causes ayant pu provoquer le départ des religionnaires.

L'île de Ré, située en face de la place forte huguenote de La Rochelle, a subi très tôt son influence en matière de religion. Une partie de la population rhétaise, surtout celle de Saint-Martin, a adhéré aux idées de la religion réformée dès le $\mathrm{XvI}^{\mathrm{e}}$ siècle et a joué un rôle de premier plan dans l'établissement des premiers lieux de culte. Il apparaît que, dès 1545, 500 Rhétais ${ }^{83}$ étaient traduits en justice pour "hérésie», et une centaine d'entre eux ont eu leur procès instruit à La Rochelle. Mais cela ne sonna pas le glas de la Réforme dans l'île de Ré puisqu'en 1558, un consistoire était établi à La Rochelle et son fondateur, Richer, organisa l'Église de Ré qui accueillit son premier pasteur, Germain Chauveton, en mai 1560.

La pacification d'Amboise, en 1563, permit aux Rhétais d'acquérir un premier lieu de culte situé à Saint-Martin dans la maison de Henrye de la Davière, veuve Ogier, qui englobait l'ancienne chapelle Saint-Clair ${ }^{84}$. La même année, le roi Charles Ix invita le gouverneur de La Rochelle à favoriser sur l'île le baptême des enfants de protestants, prenant en considération «l'incommodité et le danger de naufrage que couraient les enfants qu'il fallait porter à baptiser à La Rochelle en passant 4 ou 5 heures de $\operatorname{mer}^{85}$ ». Les protestants mirent ensuite à profit l'édit de Nantes pour construire leurs temples. Le premier fut construit à Saint-Martin en 1599, suivi par ceux d'Ars et de La Flotte.

Plusieurs sources permettent d'estimer l'importance des huguenots dans l'île de Ré et plus particulièrement à Saint-Martin. Si l'on se rapporte aux contrats de mariage relevés à Saint-Martin, $22 \%$ concernent des mariages protestants pour la période comprise entre 1620 et 1685 . Perouas confirme qu'en 1663 le nombre de religionnaires rhétais oscillait entre 1900 et 2400 , soit 13 à $15 \%$ de la population de l'île ${ }^{86}$. Il précise que 1100

83. Pierre Dez, Histoire des protestants et de l'église Réformée de l'île de Ré (La Rochelle, Pijollet, 1926), 2.

84. Ibid., 7.

85. Monique Jambut, L'isle du Roy sous Louis XIII. De la réforme aux guerres de religion (Châteauroux, 1984), 31.

86. Louis Perouas, op. cit., 132. Chiffres fournis par les visites de Henry de Laval en juin 1663. 
à 1300 huguenots, donc plus de la moitié d'entre eux, résidaient à SaintMartin. Ainsi, en dépit d'un pourcentage qui faisait d'eux une minorité dans l'île, cette concentration dans le bourg le plus important de l'île leur assurait une position solide. Pierre Dez, qui a comptabilisé les baptêmes et mariages protestants dans l'île, va plus loin en supposant une population protestante de 3000 à 4000 âmes pour toute l'île ${ }^{87}$.

Il n'y a donc rien d'étonnant à retrouver des émigrants issus des sphères protestantes de Saint-Martin, d'autant que leurs familles appartenaient au milieu des négociants, commerçants et militaires essentiellement huguenot. Ainsi, Jacques Baudoin était le fils de Solon Baudoin, un important négociant huguenot de Saint-Martin. L'artisan tonnelier protestant Isaac Dodin partit avec une de ses filles, Hélène, et était présent à son contrat de mariage à Montréal en 1664. René, le père de Barbe Ménard, était un protestant rochelais tailleur d'habits. Enfin, le sieur Jean Geoffroy, dit "Maizon Blanche», le père supposé de Nicolas Geoffroy, serait un soldat au fort de La Prée, près de Saint-Martin.

Les familles de ces émigrants étaient parfois des réformés depuis plusieurs générations, comme c'était le cas des familles Baudoin et Ménard. Les Baudoin blasonnant "de sable au lion lampassé d'azur au franc-canton senestre d'hermine " étaient calvinistes depuis au moins la fin du XvI ${ }^{\mathrm{e}}$ siècle avec Jacques Baudoin, sénéchal de l'île de Ré et sa femme Anne Collard, les grands-parents paternels de l'émigrant Jacques Baudoin. Pareillement, les grands-parents paternels et maternels de Barbe Ménard étaient des protestants rochelais ${ }^{88}$.

Si le culte réformé à l'île de Ré paraît ancien et bien implanté, a-t-il donné lieu à des persécutions et tracasseries qui auraient pu pousser au départ certains protestants martinais? Le développement du culte protestant à Saint-Martin ne semble pas, en effet, s'être déroulé sans heurts aux $\mathrm{XVI}^{\mathrm{e}}$ et XVII ${ }^{\mathrm{e}}$ siècles, notamment entre les années 1628 et 1685 qui nous intéressent plus particulièrement ${ }^{89}$. Par exemple, en février 1630, «une ordonnance invite toutes les églises réformées du gouvernement de $\mathrm{La}$ Rochelle à faire valoir leurs titres d'existence. Or, ceux de l'église de Ré seront égarés par l'intendant Coignet de la Thuilerie qui, le 20 mars 1630,

87. Ibid.

88. ADCM, mariage de Pierre Veillon et Renée Girard, 29 mai 1610, registres du temple calviniste de La Rochelle.

89. Voir Claire Lambert, Une contribution de l'île de Ré au peuplement de la Nouvelle-France : la paroisse de Saint-Martin au XVII ${ }^{e}$ siècle, op. cit., 43-49. 
suspend l'exercice du culte reformé dans l'île $\mathrm{e}^{90}$.» Perouas y voit la volonté de briser l'Église calviniste rhétaise solidement implantée ${ }^{91}$. Le 18 mars 1630, une émeute fomentée par les capucins entraîna la démolition du temple d'Ars. Le culte, rétabli en 1648, ne put être maintenu qu'à SaintMartin. Mais les protestants étaient privés de leurs charges et ceux qui n'étaient pas originaires de l'île furent expulsés, comme ce fut également le cas à La Rochelle.

En 1663, une vérification fut exigée concernant les titres des églises. Il s'ensuivit une querelle juridique pendant laquelle Colbert de Terron interdit le culte à Saint-Martin et les catholiques en profitèrent pour piller le temple. Les discussions restant sans résultat, la question fut soumise au Conseil d'État qui autorisa en mars 1671, huit ans après le début du litige, l'exercice du culte à Saint-Martin, mais en maintint l'interdiction pour Ars et La Flotte.

Ces mesures semblent avoir encouragé l'émigration, même s'il est douteux que les départs vers la Nouvelle-France aient pu être le résultat des guerres et des tracasseries, les huguenots n'ayant pas la possibilité d'y former officiellement une communauté ${ }^{92}$. Déjà, en 1627 , quelques protestants rhétais avaient préféré s'expatrier en Angleterre. En 1664, de nombreuses familles huguenotes de La Rochelle et de Saint-Martin migrèrent au Massachusetts. À partir de 1675 , ce mouvement reprit et s'accentua jusqu'à la révocation de l'édit de Nantes en 1685 .

Les sources martinaises ne permettent pas de mesurer le rôle joué par l'appartenance à la religion réformée dans le départ des quelques protestants de Saint-Martin vers la vallée du Saint-Laurent. En tout cas, ceux-ci semblent bien avoir renié leur foi, comme Barbe Ménard et Marie Jalais se mariant toutes deux, le 26 août 1669 à l'île d'Orléans, selon la religion catholique, ainsi que Jacques Baudoin et Nicolas Geoffroy abjurant dès leur arrivée en 1664 et 1665 .

\section{CONCLUSION}

Les résultats de cette étude se rapportent à différents éléments ayant pu influencer la décision de quitter le sol rhétais en direction du Canada au XvII ${ }^{\mathrm{e}}$ siècle. Dans la majorité des cas, les émigrants et leurs familles étaient de

90. Pierre Dez, op. cit., 34.

91. Louis Perouas, op. cit., 132.

92. La politique était en effet «d'empêcher la présence d'une Église protestante constituée, mais nullement d'empêcher l'émigration individuelle des huguenots", Robert Larin, op. cit., 139. 
souche, du moins martinaise, sinon rhétaise. Ils avaient donc baigné, avant leur départ, dans un environnement social, économique et religieux spécifique, marqué notamment par la proximité avec le port de La Rochelle.

En premier lieu, la société martinaise semble profondément conditionnée par la monoculture de la vigne; la possession pérenne de la terre à l'intérieur des familles et la transmission de ce patrimoine depuis souvent plusieurs générations créaient un véritable attachement à la vigne. De plus, il ne s'agissait pas de familles vivant dans la misère, comme le démontre l'étude des apports au mariage. Même si l'exploitation était le plus souvent de taille réduite et morcelée, les parcelles présentaient en général une superficie suffisante pour en vivre, d'autant que la pratique d'un travail annexe pouvait assurer des revenus complémentaires.

Mais, dans le but d'éviter un morcellement croissant de l'exploitation à la suite d'un partage égalitaire strict en contexte de saturation du terroir, les familles ont mis en place des stratégies visant à sa préservation. Ainsi la formation d'alliances matrimoniales au sein de plusieurs familles autorisait des remembrements occasionnels. Ces alliances endogames permettaient d'agrandir l'exploitation grâce à l'apport du conjoint doté de pièces de vigne par ses parents, originaires également de l'île. Ensuite, les familles pouvaient contourner le partage égalitaire strict, prescrit par la coutume, par le moyen de rachats des parts d'un héritier à ses autres cohéritiers moyennant une compensation financière ${ }^{93}$.

Cette situation pouvait encourager le départ de certains, les émigrants s'excluant alors eux-mêmes du partage successoral dans le but de préserver le patrimoine familial. Cette possibilité était bien réelle puisque la transmission des biens prenait en compte le départ préalable des héritiers présomptifs. En effet, en aucun cas les émigrants partis au Canada n'étaient présents ou même cités lors des partages après décès qu'il nous a été donné d'observer. Ne possédant pas déjà, pour la plupart, de biens fonciers dans la paroisse de Saint-Martin avant le départ, les émigrants subissaient dans les faits une exclusion du patrimoine familial pour cause de non-résidence dans la paroisse.

Le remariage éventuel d'un des parents compliquait encore davantage la succession. Dans ce cas, le parent survivant dotait en espèces les enfants de son précédent mariage de leurs droits par rapport au parent décédé. Le futur émigrant possédait alors quelque argent pour s'expatrier.

93. Voir Claire Lambert, Une contribution de l'île de Ré au peuplement de la Nouvelle-France : la paroisse de Saint-Martin au XVII ${ }^{e}$ siècle, op. cit., 70. 
En second lieu, les futurs émigrants ont pu être sensibilisés aux voyages au long cours par suite de l'activité portuaire importante de Saint-Martin avec La Rochelle et de nombreux pays européens ou outre-Atlantique. De la même manière, certaines expériences migratoires vécues par des membres de leurs familles, notamment aux Antilles, ont pu les familiariser avec la migration.

L'influence de la ville de La Rochelle auprès de l'île de Ré n'était pas seulement économique, mais également idéologique, ce bastion de la Réforme ayant véhiculé les idées protestantes auxquelles a adhéré une partie de la population martinaise, dont quelques émigrants pour la colonie. Cependant, la Nouvelle-France ne faisait pas partie des pays du Refuge et le roi n'encourageait pas le départ des protestants et la formation d'une communauté huguenote dans ses colonies. Si un certain nombre de religionnaires s'embarquèrent pour la Nouvelle-France, il n'y eut jamais de communauté organisée et beaucoup abjurèrent à leur arrivée ou se comportèrent en parfaits catholiques.

Enfin, même si ces départs paraissent à première vue dénués de rapports entre eux, quelques pistes permettent de corriger cette impression première puisque la majorité des départs se concentrèrent sur une période de seulement six ans, entre 1663 et 1669 , et étaient constitués de petits groupes unis par des liens familiaux et amicaux. Les émigrants martinais se rapprochaient également par leur origine sociale, étant généralement tous issus du milieu viticole. Cependant la migration n'était pas homogène sur tous les plans, englobant aussi bien des militaires, des engagés, des Filles du roi que des passagers libres.

Au terme de cette étude, plusieurs questions restent en suspens, auxquelles l'avenir de la recherche apportera peut-être des réponses. Par exemple, le rôle de La Rochelle comme ville intermédiaire entre les campagnes environnantes et les colonies atlantiques mériterait d'être creusé, grâce à une exploitation systématique de ses registres paroissiaux et actes notariés. Les traces éventuelles qu’y ont laissées les futurs émigrants pourraient nous éclairer sur les processus de recrutement et sur l'articulation entre un passé métropolitain connu le plus souvent à travers les membres de leurs familles et un avenir colonial qui comporte encore de vastes zones d'ombre. Il reste donc à élargir l'enquête prosopographique déjà amorcée, par la multiplication des essais biographiques d'émigrants et par le suivi dans l'espace régional et national des cas étudiés. L'analyse des trajectoires familiales pré et postmigratoires est également porteuse d'espoirs de recherche sur la signification sociale de la migration : en quoi le destin des 
branches séparées d'une même famille - l'une restée en France, l'autre implantée au Canada - était-il différent? La démographie historique a déjà fourni quelques éléments de réponse à cette question, sur le terrain des comportements démographiques différentiels ${ }^{94}$, mais l'histoire sociale ne s'est guère penchée, ou seulement de façon très parcellaire, sur la transmission des comportements sociaux ou culturels (en fonction des solidarités d'origines et des pratiques langagières, religieuses, architecturales, criminelles, etc.). L'histoire socioculturelle des immigrants, vue du milieu d'origine français au milieu d'acculturation canadien, n'est pas encore écrite. 